\title{
Belgeo
}

Revue belge de géographie

\section{La construction épistémologique de l'urbanisme en Belgique}

Epistemological construction of urbanism in Belgium

\section{Geoffrey Grulois}

\section{(2) OpenEdition}

\section{Journals}

Édition électronique

URL : http://journals.openedition.org/belgeo/6329

DOI : $10.4000 /$ belgeo.6329

ISSN : 2294-9135

Éditeur :

National Committee of Geography of Belgium, Société Royale Belge de Géographie

\section{Édition imprimée}

Date de publication : 30 juin 2011

Pagination : 5-16

ISSN : 1377-2368

\section{Référence électronique}

Geoffrey Grulois, "La construction épistémologique de l'urbanisme en Belgique », Belgeo [En ligne],

1-2 | 2011, mis en ligne le 15 décembre 2012, consulté le 20 avril 2019. URL : http://

journals.openedition.org/belgeo/6329; DOI : 10.4000/belgeo.6329

Ce document a été généré automatiquement le 20 avril 2019.

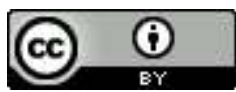

Belgeo est mis à disposition selon les termes de la licence Creative Commons Attribution 4.0 International. 


\title{
La construction épistémologique de l'urbanisme en Belgique
}

Epistemological construction of urbanism in Belgium

\author{
Geoffrey Grulois
}

\section{Introduction}

Dans les années soixante et septante, Françoise Choay construit une historiographie critique de l'urbanisme qui questionne cette discipline apparue vers la fin du XIXe siècle pour répondre aux problèmes de l'aménagement des sociétés industrielles (Choay, 1965, 1980). Pour cette philosophe, les théories d'urbanisme légitiment des modèles normatifs et utopiques qui créent une rupture spatiale, sociale et temporelle dans le processus de fabrication de la ville (Choay, 1965, p. $15 ; 1980$, pp. 290-333).

2 L'urbanisme "culturaliste" sous-tend un regard nostalgique sur l'unité organique de la ville médiévale (Choay, 1965, pp. 21-26, pp. 41-46). La déconcentration de la grande ville suivant le modèle des "cités-jardins" en constitue l'archétype défini par le réformiste anglais Ebenezer Howard à la fin du XIXe siècle (Howard, 1898). A l'opposé, le modèle "progressiste" est conditionné par la foi dans la science et la technique comme moyen de réformer la société par l'espace (Choay, 1965, pp. 16-20, pp. 31-41). La ville concentrée, bâtie en hauteur et fonctionnellement zonée forme le modèle universel imposé par l'architecte suisse Le Corbusier à partir des années vingt (Le Corbusier, 1920).

Les travaux sur l'histoire de l'urbanisme en Belgique identifient l'émergence de cette discipline autour de la Première Guerre mondiale (Smets, 1977, 1985 ; Uyttenhove, 1985 ; Zampa, 1999). Ils ont souligné le rôle joué par l'ingénieur Raphaël Verwilghen dans l'importation du modèle de la cité-jardin et des outils du town-planning anglais (Smets, 1977, pp.106-114 ; Stynen, 1979, pp. 16-41 ; Smets, 1985, pp. 77-78 ; Uyttenhove, 1985, pp. 36-59 ; Zampa, 1999, pp. 61-149). Sur le plan théorique, ces travaux ont mis en avant l'importance des Préliminaires d'Art Civique de l'architecte paysagiste Louis Van der Swaelmen (Van der Swaelmen, 1915 ; Smets, 1977, pp. 92-94 ; Stynen, 1979, pp. 69-79 ; 
Uyttenhove, 1985, pp. 51-54). Ils montrent que ces deux protagonistes vont prolonger leur réflexion après la Première Guerre mondiale en fondant la Société des Urbanistes Belge (SUB) et la revue La Cité.

4 Malgré l'engagement de ces deux protagonistes, la littérature secondaire remarque le manque de postérité de l'oeuvre théorique de Van der Swaelmen (Stynen, 1979, p. 79) et la disparition abrupte du modèle de la cité-jardin avec la domination univoque des théories fonctionnalistes de Le Corbusier et des Congrès Internationaux d'Architecture Moderne à partir de la fin des années vingt (Smets, 1977, pp. 140-161; Uyttenhove, 2003, p.401). Implicitement l'historiographie de l'urbanisme en Belgique confirme la dichotomie des modèles proposée par Choay en identifiant le basculement du modèle culturaliste de la cité-jardin vers le modèle progressiste de Le Corbusier dans la deuxième moitié des années vingt. Or le climat idéologique des années septante et quatre-vingt contribue à stigmatiser l'urbanisme progressiste et fonctionnaliste parce qu'il "détruit la ville en réponse aux exigences du développement des structures industrielles $d u$ capitalisme privé et public" (Barey, 1980, p.17). Choay reconnaît que ses travaux d'histoire et d'épistémologie de l'urbanisme moderne ont visé à "dénoncer l'imposture d'une discipline qui, dans une période de construction fiévreuse, imposait son autorité sans conditions" (Choay, 1980, p. 16).

Dans cet article nous cherchons à questionner les fondements épistémologiques de l'urbanisme en Belgique en retraçant le cheminement intellectuel construit par les fondateurs de la Société des Urbanistes Belges et de la section belge des Congrès Internationaux d'Architecture Moderne depuis la Première Guerre mondiale jusqu'aux années cinquante. A la différence des recherches antérieures qui ont avant tout cherché à montrer l'influence des modèles antinomiques de Howard et de Le Corbusier, nous chercherons à identifier les spécificités épistémologiques de l'urbanisme en Belgique.

\section{Le vitalisme urbain}

Elles sont une espèce de transposition où en un seul rythme s'expriment des états d'âmes, d'esprits, de moeurs. Elles sont ces plantes vivaces qui plongent leurs racines, les multiplient, les dispersent, puisent leur existence dans tous les terrains humains et, ensuite, en leurs organismes robustes condensent les éléments différents, les soumettent à un travail d'assimilation et les confondent alors à une même substance, en un même fruit (De Ligne, 1918, pp. 41-42).

6 Les travaux de Smets (1985, pp. 75-83), Uyttenhove (1985, pp. 36-42, pp. 57-59) et Zampa (1999, pp. 61-105) insistent sur le rôle joué par l'ingénieur Raphaël Verwilghen dans la définition du premier Arrêté-loi sur l'urbanisme de 1915. Or, cet Arrêté-loi dont le contenu normatif est inspiré par le town-planning anglais et le modèle de la cité-jardin, n'aura que peu d'influence sur la reconstruction après la Première Guerre mondiale (Smets, 1985, pp.85-96; Uyttenhove, 1985, p. 67). Nous pensons qu'il occulte une réflexion sur les fondements du processus de fabrication de la ville en Belgique qui est mise en place au même moment par un auteur dont la contribution a été systématiquement ignorée par l'historiographie de l'urbanisme en Belgique: Jean De Ligne.

7 Pendant la guerre, cet architecte diplômé de l'Académie des Beaux-Arts de Bruxelles entreprend l'étude historique d'une ville belge en s'inspirant des travaux de l'historien belge Henri Pirenne (De Ligne, 1918). Dès la fin du XIX ${ }^{e}$ siècle, Pirenne avait entamé une 
histoire des villes des Pays-Bas méridionaux qui va créer sa réputation parmi les médiévistes et les spécialistes de l'histoire économique (Pirenne, 1893 \& 1898 ; Ganshof, 1959).

Ypres, essai sur sa formation et sa reconstruction cherche à mettre en avant l'évolution des formes urbaines en fonction des "courants humains" et du "milieu" qui façonnent le territoire belge (De Ligne, 1918). Pour De Ligne, la ville est un corps vivant à l'image de son être social et géographique, elle constitue une unité organique en interaction avec le milieu naturel et humain. Ces formes architecturales "sont le produit d'une tendance, d'une aspiration, de l'idéal de l'époque dans laquelle elles sont formées" (ibid., p. 41). Dans la conception de De Ligne, la ville évolue continuellement dans un équilibre environnemental avec l'homme et son milieu.

9 L'architecture est déterminée par les conditions géographiques, économiques et sociales de l'époque, elle "ne subit aucune influence d'un art de construire local d'époque antérieure, fut essentiellement elle-même, personnelle, soumise seulement à l'imagination simple, robuste, primitive, des constructeurs de la commune d'Ypres" (ibid., p. 45). L'inspiration civique de ces constructeurs perpétue l'évolution continue de la cité : "l'âme de la ville par des corps en communion avec cette âme; avec cette âme que ce passé avait perpétué de corps en corps par-delà la mort successive de ces êtres" (ibid., 1918, p. 51).

10 De Ligne construit une approche de l'urbain que le philosophe Georges Canguilhem qualifie de "vitaliste" en opposition avec le modèle mécaniste des sciences modernes (Canguilhem, 1965, pp. 83-100). Dans le prolongement des travaux de Bergson, De Ligne envisage la ville comme une totalité évoluant de manière continue, irréductible à une décomposition mécaniste ou un modèle normatif (Bergson, 1907). C'est pourquoi, à la différence de l'ingénieur Verwilghen, De Ligne ne peut s'engager dans une démarche scientifique pour déterminer les outils normatifs de l'urbanisme. Pour lui, seule l'existence empirique, l'étude historique, et l'intuition créatrice permettent de guider le constructeur dans le processus de transformation de la ville.

11 Or c'est aussi ce principe vitaliste que La Société des Urbanistes Belges (SUB), fondée par De Ligne, Van der Swaelmen et Verwilghen, entend mettre en avant dans l'après-guerre (Bodson et al., 1919 ;, Grulois, 2011). Comme le précise un de ses fondateurs, les urbanistes veulent avant tout répondre "au sens de la vie" alors que les techniciens héritiers de l'Art urbain du XIXe siècle "tentent de figer la vie de la rue et de la mécaniser" (Bodson, 1919, p. 61). La démarche vitaliste de certains urbanistes belges s'oppose aux modèles normatifs dénoncés par Choay en cherchant à inscrire l'urbanisme dans l'exploration du processus de fabrication de l'urbain.

\section{La tension épistémologique}

Initié à la Loi historique du développement de la Cité considérée, pénétré des répercussions du Facteur géographique sur les dispositions existantes ou à prévoir, respectueux des Vestiges archéologiques, attentif au battement du pouls de la Cité fonctionnelle, il conçoit entre tous ces facteurs des concordances harmonieuses (Van der Swaelmen, 1916, p. 79).

12 A la lumière de l'approche vitaliste énoncée par De Ligne, nous pouvons tenter de comprendre la démarche théorique des Préliminaires d'Art Civique de Van der Swaelmen 
(1916). Dans cet ouvrage, Van Der Swaelmen reconnait le processus d'évolution naturel des cités pré-industrielles :

D'abord celle pendant laquelle l'homme, ignorant de ce que nous nommons les Lois de la Nature, qui le guident, et très obscurément conscient des mobiles qui le poussent, obéit aux suggestions de son instinct, qui le conduisent toujours plus en avant dans une direction commune, chronologiquement, continue, orientée et propulsée par un déterminisme absolu (ibid., p. 10).

13 Ces cités anciennes se sont formées spontanément mais l'individualisme engendré par la modernité tend à rompre leur unité organique. Elle définit une deuxième époque.

Celle que l'homme inaugure lorsqu'échappant complètement à la conduite de l'instinct, il délaisse l'"esprit de la ruche" - inévitable corollaire d'être devenu trop conscient de ses mobiles individuels - sans qu'il se soit élevé encore à une conscience collective, à une connaissance généralisée des phénomènes fondamentaux, capable de suppléer au fléchissement de l'instinct (ibid., p. 17).

14 Van der Swaelmen envisage l'Art civique comme une discipline dont l'objectif est de prolonger la vitalité et l'unité de l' “organisme vivant collectif” (ibid., p. 30). Cette discipline se construit à partir d'une "science de l'observation" qui permet d'identifier empiriquement les lois du développement urbain (ibid., p. 19). Pour Van der Swaelmen,

il est toujours possible de dégager de l'examen des extensions successives d'une cité la Loi interne suivant laquelle s'effectue le Développement extensif centrifuge de cette Cité sous l'empire de circonstances avant tout géographiques, chronologiques et économiques (ibid., p. 42).

15 L'Art civique doit étudier le développement accéléré des villes depuis le milieu du XIX siècle qui tend à rompre son unité organique en dissociant artificiellement certaines fonctions (ibid., pp. 43-54) (figure 1). A la différence des systèmes normatifs dénoncés par Choay (1980, pp. 303-314), Van der Swaelmen construit une démarche inductive basée sur l'observation empirique du processus d'évolution de la ville. En ce sens il se rapproche des théories développées par le biologiste Patrick Geddes dans son ouvrage Cities in Evolution sans pour autant en avoir reçu l'influence directe (Geddes, 1915 ; Choay, 1965, pp. 58-64 ; Stynen, 1979, p. 70 ; Uyttenhove, 1985, p. 50). 
Figure 1. Van der Swaelmen. (1916), La génération spontanée d'une ville : Anatomie et physiologie de la Cité, Préliminaires d'Art civique mis en relation avec le cas clinique de la Belgique, Leyde, A.W. Sijthoff, p. 38.

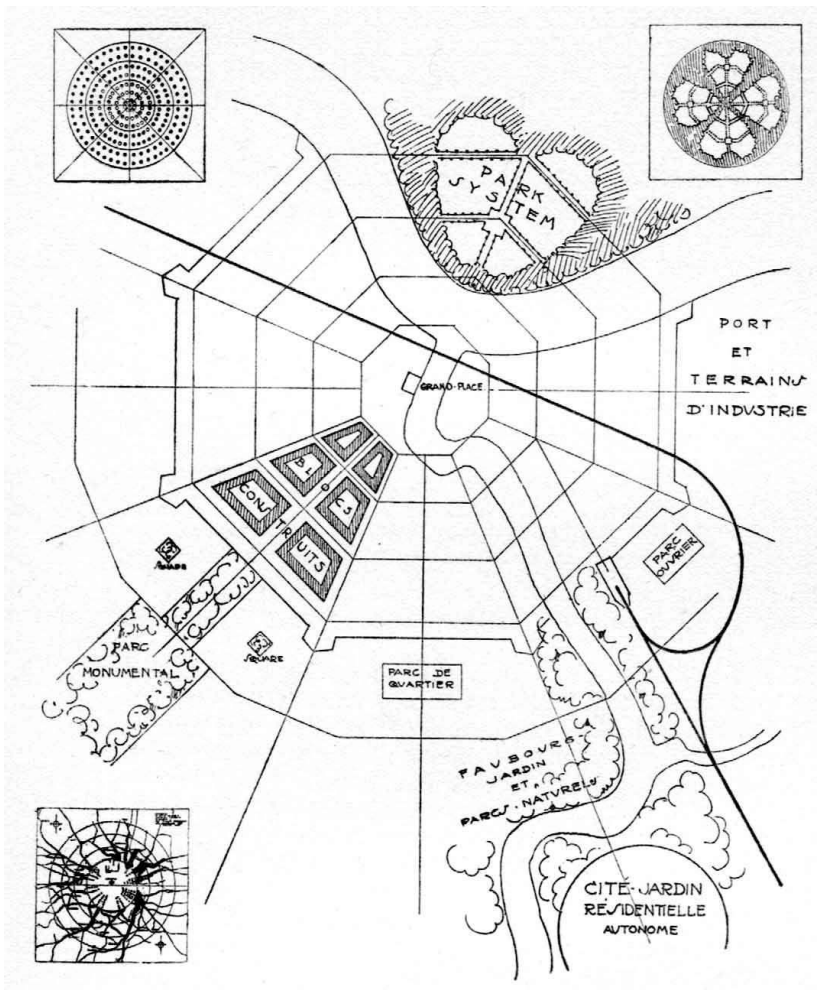

Mais à la différence de Geddes et De Ligne, Van der Swaelmen cherche à objectiver et normaliser le processus d'observation de l'urbain. C'est pourquoi il importe la technique du civic development survey standardisée par le town planner anglais Lanchester (ibid., pp.141-163). Cette technique d'enquête contribue à réduire le processus de description empirique de l'urbain au moyen d'une décomposition mécaniste de son objet d'étude. Cette même volonté d'objectiver la discipline le conduit à imaginer une Encyclopédie des Villes et de l'Art civique afin de dresser le tableau des "standards expérimentaux universels" (Van der Swaelmen, 1916, p. 167).

Van der Swaelmen, comme Verwilghen, ne semble pas conscient de la réduction opérée par ce principe de causalité mécaniste. Pressé par la volonté de légitimer l'urbanisme comme une science de l'action, il n'a pas entendu l'avertissement formulé par Bergson, pour qui ce "déterminisme inflexible... manifeste l'interruption de l'acte créateur" (Bergson, 1907, p. 218).

Dans l'après-guerre, la volonté d'objectiver l'urbanisme contribue à dissocier le "plan régulateur" de la démarche d'observation empirique suivant un principe de décomposition mécanique. Détaché de son ancrage empirique, le plan régulateur se réduit à "l'expression graphique d'une synthèse organique d'éléments fonctionnels dont l'étude fait l'objet de la science urbanistique" (Bodson et al., 1919, p. 38). Il prolonge l'approche instrumentale initiée pendant la guerre par l'ingénieur Raphaël Verwilghen sur le "plan général d'aménagement" (Verwilghen, 1919, p. 17 ; Smets, 1985, pp. 81-86 ; Zampa, 1999, pp. 68-106). 


\section{L'agglomération industrielle}

19 Ces agglomérations provoquées par le passage de relations économiques et nourries continuellement de cette sève, allaient se développer et secréter leurs cellules urbaines ; elles allaient le faire en adaptant leur groupement structural à ce mouvement constant des échanges. Aussi allaient-elles créer et constituer des organes urbains, des artères capables de drainer, d'accrocher et d'assimiler ces courants féconds, et allaient-elles grouper leur masse urbaine, en des formes différentes suivant que les courants y convergeaient, s'y rencontraient ou bien y passaient (De Ligne, 1925, p. 120).

20 Dans les années vingt, les membres de la Société des Urbanistes Belges vont prolonger leurs réflexions méthodologiques en l'appliquant au problème de la grande ville industrielle. Dès 1924, Verwilghen dénonce la croissance libérale de la grande ville. Pour lui la ville est "une émanation du capitalisme" (Verwilghen, 1924, p. 23). Elle semble "devoir inévitablement s'étendre, comme une tache d'huile s'épand sur une feuille de papier" (ibid., p. 21). C'est en particulier le phénomène de la spéculation foncière et immobilière entretenu par les propriétaires fonciers qui "est à l'origine de la grande défectuosité des villes, défectuosité constitutionnelle" (ibid., p. 24). Pour remédier à ce développement pathologique, Verwilghen propose un schéma normatif de croissance déconcentrée en villes satellites inspiré par le modèle de Howard (ibid., pp. 30-36 ; Zampa, 1999, pp. 301-318).

21 A la même époque l'architecte suisse Le Corbusier condamne le désordre des grandes villes contemporaines et renie le modèle organique de la ville médiévale (Le Corbusier, 1925, p. 5). Pour lui, la ville n'est plus un organisme évoluant avec le milieu naturel car une ville "c'est la mainmise de l'homme sur la nature. C'est une action contre la nature" ( ibid., p. 1).

22 Comme l'a montré Choay (1965, pp. 30-46), ces deux approches normatives émergent de la volonté d'introduire une rupture spatiale et temporelle dans le processus de développement de la grande ville industrielle. A l'opposé de cette rupture, De Ligne comprend la croissance de la grande ville comme un processus d'évolution naturelle défini par les infrastructures industrielles et les moyens de transport modernes (De Ligne, 1925). Comme pour les villes médiévales, "si elles se sont élevées et développées, c'est parce que la vie constructive de leur germe avait été provoquée et entretenue par les courants favorables" (De Ligne, 1925, p. 119).

23 Au-delà du modèle culturaliste de la cité-jardin et celui de la ville dense bâtie en hauteur, De Ligne identifie un phénomène d'agglomération qui déborde les frontières communales pour s'ancrer dans le territoire belge. L'agglomération est "par ses voies de communications multipliées et amplifiées, un des plus puissants axes de l'industrie" (De Ligne, 1929, p. 104). La ville perd son "autonomie communale", elle "n'appartient plus à elle-même, elle appartient à la nation entière" (ibid., p. 103).

24 L'architecte moderniste Victor Bourgeois va chercher à étudier ce phénomène d'agglomération industrielle. Actif dans les milieux artistiques et modernistes bruxellois dès la Première Guerre mondiale, Bourgeois participe à la conférence pour l'Etude de l'Administration et de l'Urbanisation des Grandes Agglomérations organisée par l'Union des Villes et Communes belges en 1924. Elle lui révèle "le devoir, libérateur, de l'urbanisme" (Bourgeois, 1924, p. 47). La même année Bourgeois s'engage activement dans 
le processus de transformation de la SUB en Société Belge des Urbanistes et Architectes Modernistes (SBUAM).

Bourgeois semble très influencé par les conceptions urbanistiques de Le Corbusier. Il invite l'architecte suisse à Bruxelles en 1926 pour parler de la Ville Radieuse. En 1930, il organise le $3^{e}$ Congrès International d'Architecture Moderne (CIAM) à Bruxelles au cours duquel Le Corbusier prescrit le modèle des "villes en concentration, bâties en hauteur" (Le Corbusier, 1931, p. 52). Pour Le Corbusier, le développement urbain est définitivement rompu et désormais "ce sont les techniques modernes qui apportent la solution à l'urbanisation des villes" (loc. cit.). L'étude urbaine n'est plus envisagée que pour dénoncer la forme inadaptée des villes par rapport à la civilisation machiniste. Pour pouvoir appliquer le modèle idéal de la ville verticale, l'autorité doit effacer la ville existante et mobiliser son sol (ibid., pp. 55-56).

Si en apparence les projets de Bourgeois semblent très influencés par le modèle corbuséen, sa réflexion urbanistique présente des affinités importantes avec la conception vitaliste et empirique mise en avant par De Ligne. La démarche urbanistique de Bourgeois ne part pas d'un modèle idéal et déterritorialisé, elle prend appui sur l'étude du milieu urbain existant. Dans les années trente, Bourgeois lance avec Marcel Schmitz la revue Bruxelles qui rassemble les études des spécialistes issus de toutes les disciplines (s.n., 1933). Il veut constituer un véritable laboratoire de recherche pluridisciplinaire "autour duquel pourra se former l'équipe des constructeurs du Nouveau Bruxelles" (ibid., p. 3).

Son étude pour "L'Urbanisation du Grand-Bruxelles" (Bourgeois, 1932, pp.167-183) (figure 2) semble appliquer ce que De Ligne définit comme le principe exploratoire d'une "anatomie urbaine dont la connaissance nous élèvera au rang d'architectes spécialistes; spécialistes capables alors seulement de conduire la croissance constante des villes en leur assurant, dès aujourd'hui, une structure organique en fonction directe avec le milieu général dans lequel elles doivent continuer leur développement cellulaire" (De Ligne, 1925, p. 120). 
Figure 2. Bourgeois V. (1932), De la ville marchande à la ville marchande - administrative industrielle et internationale. "L'urbanisation du Grand-Bruxelles", L'Emulation, année 52, n 6 , p. 171.

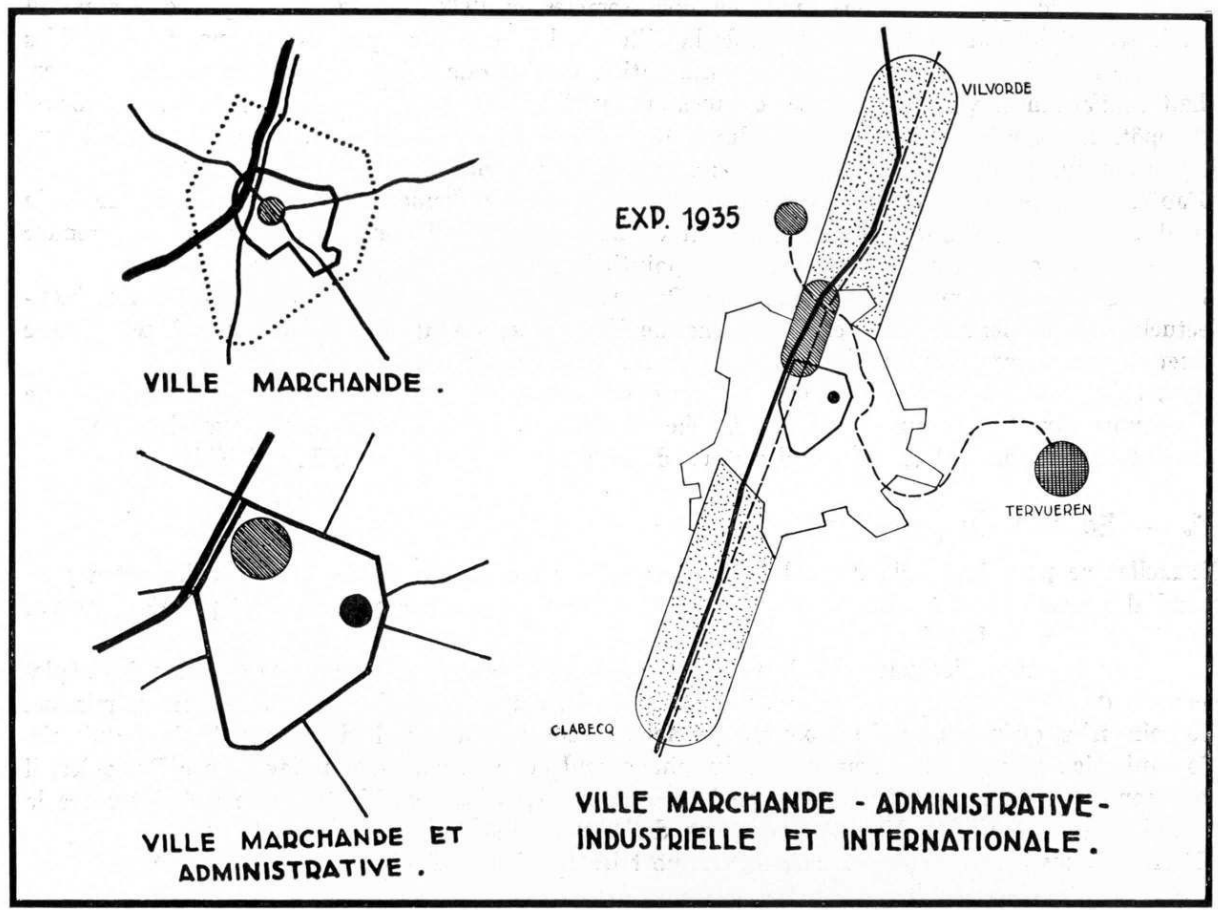

Bourgeois, comme De Ligne, reconnaît que l'unité organique de l'urbain s'est élargie bien au delà des frontières communales, mais il perçoit l'agglomération bruxelloise dans la continuité d'un processus d'adaptation à l'industrialisation du milieu. En focalisant sur le problème des infrastructures de transport moderne, Bourgeois veut mettre en avant les vecteurs d'évolution de l'agglomération.

En collaboration avec Verwilghen, Bourgeois va investiguer le problème de l'agglomération industrielle belge lors de la préparation du IV Congrès International d'Architecture Moderne (CIAM). Après le Congrès de Bruxelles de 1930, le CIAM IV veut focaliser sur le problème de la systématisation de la technique du survey et de la cartographie de la "ville fonctionnelle" (CIAM, 1932). Le Comité des CIAM demande aux sections nationales d'étudier le problème des grandes villes en appliquant les outils scientifiques et standardisés développés par l'urbaniste hollandais van Eesteren pour son analyse d'Amsterdam (ibid., pp. 2-3).

Pourtant Victor Bourgeois et Raphaël Verwilghen vont remettre en question l'approche et l'objet d'étude normatifs proposés par van Eesteren. Pour Verwilghen, qui semble avoir pris une certaine distance avec le modèle normatif de la cité-jardin,

"Il y a plus d'intérêt à étudier le processus de la formation (cristallisation) des groupements urbains en Belgique, que d'étudier quelques grandes villes isolément, celles-ci étant en train de perdre leur caractère en tant qu'unités indépendantes" (Verwilghen, 1932, p. 1).

31 Quelques mois plus tard, Verwilghen accuse la vision monumentale et décontextualisée de la ville héritée du XIX d'être à l'origine de la crise. Pour sortir de cette crise économique et épistémologique, il faut substituer le concept de "la région urbanisée" à 
celui de "la ville mondiale radio-concentrique" (Verwilghen, 1933, p. 1). Le phénomène d'agglomération linéaire qu'il identifie comme la spécificité du territoire belge, s'oppose au modèle corbuséen de la métropole concentrée et à celui de la décroissance concentrée :

Dans ce phénomène naturel de cristallisation urbaine, un exemple est typique entre tous : c'est la formation d'une agglomération continue reliant Anvers (port de mer et Métropole commerciale) à Bruxelles (Centre géographique, politique, administratif, et de plus en plus, économique du pays) avec la région industrielle de Charleroi (bassin industriel du Hainaut, prolongé à son tour par les bassins industriels du Centre et de Mons). Cette cristallisation est favorisée par, et se ramasse autour de voies de communication par eau (canal de Charleroi- Bruxelles, Bruxelles- Anvers) (Verwilghen, 1932, p. 1).

Dans la continuité de l'épistème vitaliste mis en avant par De Ligne, le phénomène d'agglomération linéaire est perçu comme un processus d'évolution continu irréductible à des composants urbains isolés.

son rapport final préparé pour le $\mathrm{IV}^{\mathrm{e}}$ Congrès, Bourgeois reprend les objections formulées par Verwilghen : "l'étude des villes n'a plus guère de signification du point de vue urbanistique : elle doit être remplacée par celle des diverses régions économiques" (Bourgeois, 1933, p. 46). Bourgeois fait dessiner des cartes de Bruxelles et Charleroi en faisant apparaître le zonage fonctionnel préconisé par les CIAM, mais il élabore également une carte régionale de l'axe Anvers- Bruxelles-Charleroi et une série de schémas d'analyse sur l'industrie dans le Hainaut (ibid., pp. 46-48). En s'intéressant à l'évolution historique de l'industrialisation dans le Hainaut il met en avant l'évolution de l'agglomération industrielle dans son milieu régional. L'exemple de Charleroi fait apparaitre un processus d'agglomération dont le développement est guidé par les infrastructures de transport et la topographie naturelle (ibid., pp. 47-48). Il incarne les spécificités du phénomène d'urbanisation belge à l'opposé des modèles normatifs de la ville concentrée, bâtie en hauteur.

La méthode d'investigation des membres de la section belge des CIAM est profondément ancrée dans la réalité territoriale de l'agglomération industrielle. Elle cherche à orienter le développement de l'agglomération en fonction de la géographie du milieu : courants humains, topographie, orientation des vents dominants, etc.

Cette méthode d'investigation s'éloigne du processus d'objectivation scientifique préconisé par les sections néerlandaises et germaniques des CIAM. L'étude d'Amsterdam réalisée par van Eesteren suggère que la conception du plan d'aménagement doit être justifiée scientifiquement par des données statistiques et des instruments cartographiques standardisés (Mumford, 2000, p. 60).

Bourgeois s'oppose à ce processus d'objectivation de l'observation empirique.

Ces rapports et ces schémas sur le développement de l'industrie dans le Hainaut démontrent la volonté de définir l'urbanisme comme une discipline de synthèse fondée sur une démarche heuristique (figure 3). Lors d'une réunion ultérieure de la section belge des CIAM au début de 1937, Bourgeois souligne une fois de plus le défaut, qui, à son avis, caractérise la façon de procéder des groupes germaniques des CIAM, c'est-à-dire de s'encombrer d'un fatras de statistiques et de documents dont l'utilité absolue n'est pas toujours prouvée en urbanisme, ce qui leur fait perdre l'esprit de synthèse. (Fitschy, 1937, p. 1). 
Figure 3. Bourgeois V. (1934), Notes sur le développement de la métallurgie dans le Hainaut, "4e Congrès International d'Architecture Moderne : Extrait du rapport sur l'urbanisme et l'architecture en Belgique", L'Emulation, année 53, n 3, p. 48.
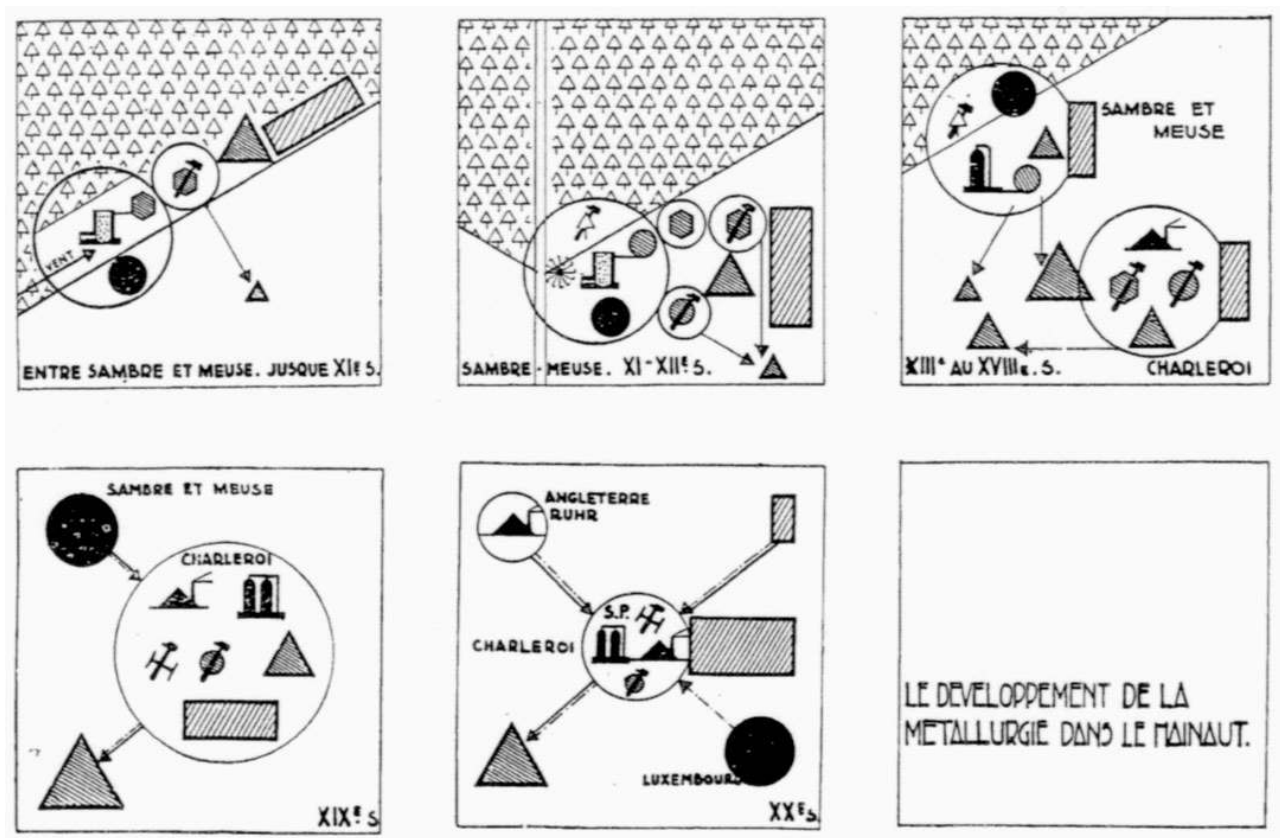

En s'opposant à l'objectivation scientifique de l'urbanisme, Bourgeois contribue à se désengager de son processus d'institutionnalisation disciplinaire. En 1935, il décide de transmettre le "flambeau" au jeune Groupe d'architectes liégeois L'Equerre en leur confiant le secrétariat de la section belge des CIAM (L’Equerre, 1935, p. 1). Dès 1936, L'Equerre organise à Liège une exposition d'Urbanisme. Elle rassemble les premiers travaux d'enquête du Comité pour l'Urbanisation de Liège créé à l'initiative de Bourgeois et du Groupe L'Equerre (Fitschy, 1936a, p. 2). Avec cette étude, le Groupe l'Equerre entend développer une recherche empirique pluridisciplinaire qui s'appuie sur une cartographie de synthèse (Fitschy, 1936b, p. 1). Dans un premier temps, elle est appliquée avec succès à l'étude de l'urbanisation de la commune de Flémalle Haute (Fitschy \& Ali, 1940).

Mais dans l'après-guerre, les enjeux de l'institutionnalisation disciplinaire contribuent à standardiser cette Technique des surveys régionaux (Fitschy et al., 1952). Comme dans le contexte de la Première Guerre mondiale, le processus de description empirique du territoire est progressivement réduit à une technique de classification standardisée qui le décompose suivant un modèle mécaniste. Comme le précise à l'époque le directeur de l'administration de l'urbanisme Victor Bure, l'approche technocratique du survey doit définir "des bases indiscutables" qui permettent aux décideurs de parler "au nom de l'opinion publique" (Fitschy et al., 1952, pp. 6-7). L'exploration du phénomène d'agglomération et du milieu régional disparait au profit d'un découpage arbitraire en secteur. Finalement, l'idée du plan régional comme outil de synthèse et de prospection est écartée au profit du plan de secteur, instrument juridique d'une législation de l'aménagement qui renie la nature évolutive du territoire en en figeant les affectations. 


\section{Conclusion} années 1910 incarne une construction épistémologique spécifique à la nature du territoire belge. Si cette construction épistémologique va subir certaines influences étrangères, en incorporant la notion de survey au town-planning anglais, elle prend avant tout sa source dans l'étude empirique du territoire belge. La démarche empirique et vitaliste des urbanistes belges s'oppose aux théories normatives dénoncées par Choay en inscrivant l'émergence de l'urbanisme dans l'exploration du processus de fabrication de l'urbain. Cette démarche a permis à ces urbanistes d'identifier le concept ouvert et non déterministe d'agglomération comme spécificité du territoire belge à l'opposé de la rupture spatiale prônée par les modèles culturalistes et progressistes décrits par Choay.

ependant la volonté de légitimer l'urbanisme comme une discipline scientifique va inciter certains urbanistes à réduire le processus d'observation et d'induction empirique suivant un principe de causalité mécanique et normatif.

construction disciplinaire de l'urbanisme en Belgique se caractérise par une tension entre des fondements vitalistes, descriptifs, empiriques et non-déterministes et la réduction de ces fondements qui résulte du processus d'institutionnalisation disciplinaire.

Malheureusement l'historiographie a contribué à faire disparaître les fondements exploratoires et non-déterministes de l'urbanisme en Belgique en focalisant sur les aspects réducteurs du processus d'institutionnalisation disciplinaire. Plus que jamais, il nous semble important de réactiver la démarche empirique et descriptive qui animait les premiers urbanistes pour nous libérer des dogmes urbanistiques (dichotomie villecampagne, etc.) et affronter les défis environnementaux posés par le territoire belge contemporain.

\section{BIBLIOGRAPHIE}

BAREY A. (1980), Déclaration de Bruxelles, Bruxelles, Editions des Archives d'Architecture Moderne.

BERGSON H. (1907), L’Evolution créatrice, Paris, Félix Alcan.

BODSON F., DE BRUYNE H., DE RIDDER A., DE LIGNE J., HOSTE H., THIRION C., PATRI Ch., VAN

AVERBEKE E., VAN DER SWAELMEN L., VAN DE VOORDE O., VERWILGHEN R. (1919), “Manifeste de la Société des Urbanistes Belges", La Cité, 1, 3, pp. 37-40.

BODSON F. (1919), “La section belge, quelques préliminaires”, La Cité, 1, 4-5, 1919, pp. 59-68.

BOURGEOIS V. (1924), “L'urbanisme vivant”, La Cité, 5, 3, pp. 45-47.

BOURGEOIS V. (1932), “L’Urbanisation du Grand-Bruxelles”, L'Emulation, 52, 6, pp. 167-183. 
BOURGEOIS V. (1933), “4 4 Congrès International d'Architecture Moderne : Extrait du rapport sur l'urbanisme et l'architecture en Belgique", L'Emulation, 53, 3, pp. 46-48.

CANGUILHEM G. (1965), La connaissance de la vie, Paris, Vrin.

CHOAY F. (1965), L'urbanisme, utopies et réalités : Une anthologie, Paris, Seuil.

CHOAY F. (1980), La Règle et le Modèle : Sur la théorie de l'architecture et de l'urbanisme, Paris, Seuil.

CIAM (1932), 4 Congrès international pour l'architecture moderne, Moscou 1932 : Indications générales et directives en vue de préparer l'exposition et la publication, archives écrites de Victor Bourgeois, dossiers CIAM, Archives d'Architecture Moderne, 4 pages.

DE LIGNE J. (1918), Ypres, essai sur sa formation et sa reconstruction, Lausanne \& Paris, Payot et Cie.

DE LIGNE J. (1925), “Des courants favorables à la formation des villes”, L’Emulation, 45, 8, pp. 119-124.

DE LIGNE J. (1929), “Le Mécanisme urbain” suite et fin, L’Emulation, 49, 12, pp. 97-106.

FITSCHY P. (1936a), Rapport du groupe belge au CIRPAC de La Sarraz de septembre 1936, archives écrites de Victor Bourgeois, dossiers CIAM, Archives d'Architecture Moderne, 2 pages.

FITSCHY P. (1936b), Lettre de Paul Fitschy à Victor Bourgeois du 17 novembre 1936. archives écrites de Bourgeois, dossiers CIAM, Archives d'Architecture Moderne, 1 page.

FITSCHY P. (1937), Procès verbal de la séance de la section belge des Congrès Internationaux d'Architecture Moderne du 26 février 1937, archives écrites de Victor Bourgeois, dossiers CIAM, Archives d'Architecture Moderne, 3 pages.

FITSCHY P., KLUTZ E., PARENT E., THIBAUX A. (1940), Le Plan d'Urbanisation de la commune de Flémalle-Haute, 1937-1940, Administration Communale de Flémalle-Haute.

FITSCHY P., KLUTZ E., PARENT E., THIBAUX A. (1952), Les Cahiers d'urbanisme $n^{\circ} 11$ : Technique des surveys régionaux, Bruxelles, Art et Technique.

GANSHOF F-L. (1959), “Pirenne, Henri”, Biographie nationale, Bruxelles, Emile Bruylant, t. 30, colonnes 671-675.

GRULOIS G. (2011) “L'urbaniste : émergence d'une figure, discours d'une élite”, Les Cahiers de La Fonderie : Les élites dans la ville, Bruxelles, La Fonderie, pp. 90-97.

HOWARD E. (1898), Tomorrow. A peaceful path to social reform, London, Swann Sonnenschein.

LE CORBUSIER (1925), Urbanisme, Paris, Grès et Cie.

LE CORBUSIER (1930), "Le parcellement du sol des villes”, Rationelle bebauungsweisen, Ergebnisse des 3. Internationalen Kongresses für Neues Bauen, Franfurt Am Main, Verlag Englebert \& Schlosser, pp. 48-57.

L'EQUERRE (1935), Lettre du Groupe L'Equerre à Victor Bourgeois du 27 août 1935, archives écrites de Victor Bourgeois, dossiers CIAM, Archives d'Architecture Moderne, 3 pages.

MUMFORD E. (2000), The CIAM discourse on urbanism, 1928-1960, Cambridge \& London, MIT Press.

PIRENNE H. (1893), “L'origine des constitutions urbaines au Moyen-Age”, Revue historique, LIII.

PIRENNE H. (1898), “Villes, marchés et marchands au Moyen âge” Revue historique, LXVII, pp. 1-12. PUTTEMANS P. (1980), “'Entre Camillo Sitte et Le Corbusier', introduction au fac-similé de Van Der Swaelmen L. (1980)“, Préliminaires d'Art civique mis en relation avec le cas clinique de la Belgique, Leyde, A.W. Sijthoff, s.p. 
SMETS M. (1977), L'avènement de la cité-jardin en Belgique : Histoire de l'habitat social en Belgique de 1830 à 1930, Bruxelles \& Liège, Pierre Mardaga.

SMETS M. (1985) "La reconstruction belge ou le passage de l'art urbain à l'urbanisme", in SMETS M. (éd.), Resurgam : La reconstruction en Belgique après 1914, Bruxelles, Crédit Communal de Belgique.

S.N. (1933), "Bruxelles, ses hommes, ses institutions, son architecture : revue de technique urbaine", Bruxelles, 1, 1, p. 3.

STYNEN H. (1979), Louis Van Der Swaelmen (1883-1929), animateur du mouvement moderne en Belgique, Bruxelles \& Liège, Mardaga.

UYTTENHOVE P. (1985), "Les efforts internationaux pour une Belgique moderne”, in SMETS M. (éd.), Resurgam : La reconstruction en Belgique après 1914, Bruxelles, Crédit Communal de Belgique.

UYTTENHOVE P. (2003), "Urbanisme en Belgique", Dictionnaire de l'architecture en Belgique de 1830 à nos jours, Bruxelles, Mercator, pp. 398-415.

VAN DER SWAELMEN L. (1916), Préliminaires d'Art civique mis en relation avec le cas clinique de la Belgique, Leyde, A.W. Sijthoff.

VAN DER SWAELMEN L. (1919), “Les sections étrangères d'Urbanisme comparé”, La Cité, 1, 4-5, pp. 69-82.

VERWILGHEN R. (1919), “Une importante réforme de notre législation en matière d'Urbanisme : l'arrêté-loi du 25 août 1915”, La Cité, 1, 1, p. 17.

VERWILGHEN R. (1924), “Les transports en fonction du plan régional”, La Cité, 5, 2, pp. 21-36.

VERWILGHEN R. (1932), Note concernant la participation belge au CIAM de 1933, archives écrites de V. Bourgeois, dossiers CIAM, Archives d'Architecture Moderne, 3 pages.

VERWILGHEN R. (1933), "L'urbanisme et la crise économique”, Conférence au Congrès de rationalisation SBUAM du 22 janvier 1933, archives écrites de H. De Koninck, dossiers CIAM, Archives d'Architecture Moderne, 1 page.

ZAMPA F. (1999), La continuité d'une illusion. L'urbanisme de Raphaël Verwilghen entre valeurs éthiques et pratiques professionnelles, thèse non publiée, KULeuven-ASRO.

\section{RÉSUMÉS}

Durant la Première Guerre mondiale, la destruction dramatique des villes belges incite des intellectuels belges à questionner la nature de la vitalité urbaine. Dans cet article nous cherchons à montrer comment ce questionnement intellectuel va conduire à la construction épistémologique de la discipline de l'urbanisme.

Dans une deuxième partie, nous découvrons que cette construction disciplinaire va être soumise à un bouleversement conceptuel qui résulte du processus d'industrialisation et d'urbanisation des villes belges dans les années vingt. L'article analyse les moyens méthodologiques que les urbanistes belges vont mettre en place pour tenter de comprendre et de déterminer l'épistème de l'agglomération industrielle.

A travers cette étude historique, nous voulons montrer l'originalité et la cohérence de la construction intellectuelle initiée par les membres de la Société des Urbanistes Belges et prolongée par ceux de la section belge des Congrès Internationaux d'Architecture Moderne. Réévaluer ces sources épistémologiques de l'urbanisme nous semble tout particulièrement pertinent pour aborder les questions environnementales et urbaines contemporaines. 
During the first World War, the dramatic destruction of Belgian cities encouraged intellectuals to question the nature of urban vitality. This paper aims to depict how this intellectual quest has led to the epistemological construction of the discipline of urbanism.

In a second part, the essay researches how this theoretical process undergoes a conceptual upheaval that result from the industrialization and urbanization of Belgian cities in the 1920s. The paper analyzes the methodology and tools drawn together by Belgian urbanists in order to define the concept of the industrial agglomeration.

Through this historical study, the paper exemplifies the originality and coherence of the theoretical background of Belgian urbanism initiated by the members of the Society of Belgian Urbanists (SUB) and the Belgian Section of the International Congresses of Modern Architecture (CIAM). To reassess this epistemological construction of urbanism seems specifically relevant in the approach to contemporary environmental and urban problems.

\section{INDEX}

Mots-clés : urbanisme, survey, histoire urbaine, épistémologie, agglomération, Société des Urbanistes Belges (SUB), section belge des Congrès Internationaux d'Architecture Moderne (CIAM)

Keywords : town planning, survey, urban history, epistemology, agglomeration, Society of Belgian Urbanists (SUB), Belgian Section of the International Congresses of Modern Architecture (CIAM)

\section{AUTEUR}

\section{GEOFFREY GRULOIS}

Faculté d'Architecture, Université Libre de Bruxelles, Geoffrey.Grulois@ulb.ac.be 